\title{
Fuhrman Grade 3
}

National Cancer Institute

\section{Source}

National Cancer Institute. Fuhrman Grade 3. NCI Thesaurus. Code C62430.

Nuclei very irregular, approximately 20 microns; nucleoli large and prominent. 